\title{
Monitoring Mental Fatigue through the analysis of Keyboard and Mouse Interaction Patterns
}

\author{
André Pimenta, Davide Carneiro, Paulo Novais, and José Neves \\ CCTC/DI - Universidade do Minho \\ Braga, Portugal \\ \{pg20189\}@alunos.uminho.pt, \\ \{dcarneiro,pjon, jneves\}di.uminho.pt
}

\begin{abstract}
In our living, we often have a sense of being tired due to a mental or physical work, plus a feeling of performance degradation even in the accomplishment of simple tasks. However, these mental states are often not consciously felt or are ignored, an attitude that may result in human failures, errors and even in the occurrence of health problems or on a decrease in the quality of life. States of fatigue may be detected with a close monitoring of some indicators, such as productivity, performance or even the health states. In this work it is proposed a model and a prototype to detect and monitor fatigue based on some of these items. We focus specifically on mental fatigue, a key factor in an individual's performance. With this approach we aim to develop leisure and work context-aware environments that may improve the quality of life and the individual performance of any human being.
\end{abstract}

Keywords: Mental Fatigue, Monitoring, Fatigue Detection, Behavioral Biometric

\section{Introduction}

Fatigue is regarded as one of the main causes of human error. Many times its symptoms are ignored, as well as its importance for a good mental and physical condition, elementary for human performance and health $[1,2]$. The activity of driving a vehicle is a good example of the importance of fatigue in our activities or tasks, in which small errors can often and easily have a significant impact on human lives. This is even more significant in high-risk jobs such as aviation, transportation, aerospace, military or medicine, in which individuals routinely operate complex systems with a high degree of responsibility $[2,3]$. The study of this topic is thus decisive as the continuous disregard of the effects of fatigue, seen frequently as normal consequences of our busy lifestyle, may end and up affecting one's quality of life, health or even lost of life.

Fatigue is however a very subjective concept and difficult to define from a scientific point of view. It may be seen as a combination of symptoms that include loss in performance (e.g. attention loss, slow reaction to a particular event, or 
low performance in tasks to which the individual has the necessary skills), and subjective feelings of drowsiness and tiredness. From an abstract point of view, fatigue may be seen as two-dimensional, namely mental and physical. Despite the frequent inter-dependence of these two dimensions, they may be addressed independently [2].

In this work it is detailed a monitoring system for mental fatigue. It intends to detect different mental fatigue states of an individual in a non-invasive and transparent way. The system will analyze a set of features that stem from the individual's use of the computer, namely from the mouse and the keyboard. The system goes through a prior learning phase in which the behavior of an individual while using the computer without fatigue is studied. Later, it classifies its level of mental fatigue by quantifying changes in the individual's behavior. This approach will open the door to the development of better and intelligent working environments, that may be aware of their users' mental states and take actions towards the improvement of quality of life and their performance on tasks in which they are engaged.

\section{Mental Fatigue Detection}

Fatigue is a non-specific symptom. It may be estimated or detected from multiple sources, including the profile of the individual (e.g. age, gender, professional occupation, consumption of alcohol or drugs), performance and precision indicators (e.g. mouse click/movement precision or tasks delivered), or attention span (e.g. time spent on a particular task versus the time spent in other non-related tasks) [4].

The user's profile provides valuable information with respect to the potential level of fatigue [5]. It can be seen as a predicted base level of fatigue in the sense that it establishes a baseline, according to the lifestyle of the individual. These aspects have been thoroughly studied, mostly by psychologists, and encompass:

- Age - Defines de mental age of the individual. It is crucial to understand the expected cognitive abilities of the individual, which may have a tendency to degrade over time.

- Gender - The mental states are different between men and women.

- Professional occupation - Important to understand possible causes of mental fatigue, or to foil false states, since many occupations are intrinsically more tiresome or exhausting than others.

- Consumption of alcohol and drugs - The use of certain substances for short or prolonged periods of time may cause dependencies and other effects that lead to a state of mental fatigue.

Mental performance is generally seen as the combination of the speed and success/accuracy of an individual when carrying out a task. It is improved when the individual has plain use of his cognitive skills and decreased when these skills are somehow impaired or diminished [6]. Cognitive skills may include: 
- Memory - The use of our memory includes all the processes related to encoding, storage and retrieval of information in our brain. Memory loss and loss of performance in accessing memory is clearly linked to mental fatigue, besides the factor of deteriorating with age.

- Reaction time - The reaction time is the timespan between the occurrence of a stimulus and the response of our body or mind. Responses may vary from a thought or a change in the mental state to a physical movement or an alteration in our physiology. Independently of the nature of the stimulus and the response, slow reaction times are usually associated with mental fatigue.

- Concentration - Concentration is the cognitive process of selectively focusing on one aspect of the environment while ignoring the others. The loss of concentration may be caused by external factors and may sometimes even be desirable (e.g. a sudden potentially hazardous event that must be analyzed before continuing with a given task). However, we frequently loose interest or focus in our tasks, at a rate that increases with the increase of the mental fatigue.

- Accuracy and precision - Accuracy and precision may be seen as the achievement of results that are within the expected quality and timeframe for a given individual, given his cognitive and physical skills. It also represents the absence of unexpected errors. This is among the factors that are more easily observable and measured while, at the same time, closely related to fatigue.

Mental fatigue is also affected by a number of other external factors. They may or may not be directly related to the individual's sphere, thus adding to the complexity of their comprehension and study $[5,7]$ :

- Mood - The mood of the individual may influence decisively his/her the mental state, with a particular effect on his/her motivation to work. Although tired, the individual may overcome (even if only temporarily) the effects of fatigue with a positive mood and motivation.

- Stress - Stress may be defined as the set of responses of the individual's mind or body to external stimuli, allowing the individual to adapt to the dynamic requirements of the environment. These processes of adaptation, however, require an additional effort from the brain which, when prolonged over long or intense periods of time, will result in mental fatigue.

- Sleepiness - Sleepiness is often mistaken for mental fatigue or generalized as such. The difference exists and must be pointed out. However, the mistake is understandable since sleepiness is a symptom that is strongly connected to mental fatigue: it is one of the ways our brain uses to tell us that he is running out of resources. It often results in a general loss of the individual vitality.

\subsection{Indicators of Mental Fatigue}

The study of mental fatigue, including its causes and symptoms, is traditionally supported by self-reporting mechanisms (generally questionnaires) or, more recently, through the use of physiological sensors. The first approach, related to 
Psychology, has a certain number of disadvantages: (1) people often lie or hide truths in questionnaires; (2) people are afraid or unwilling to answer correctly; (3) people frequently have wrong and subjective perceptions of their symptoms; (4) questionnaires are often hard to define correctly, with inaccurate questions or answers. The second approach, followed by Medicine, has the advantage of being more accurate. However, this has its price on the invasive and intrusive nature of the physiological sensors used, which may even influence the variables of the study $[8,9]$.

In order to study fatigue we are thus following an approach already validated in the study of stress [10,11]: analyzing changes in the behavior of the user while within a given technologically empowered environment, in a non-intrusive and non-invasive way.

This results in an environment that may adjust, in real-time, to significant changes in its context, with context being defined by the level of fatigue of the individuals. The detection and classification of fatigue will be based on the collection of data about behavioral biometrics, specifically keystroke and mouse dynamics. Following this approach it is possible to collect data that will allow to discover behavioral patterns of interaction with the keyboard and mouse, in a non-intrusive and dynamic way. Particularly, the following features are considered:

- Keydown Time - time spent between the key down and the key up events;

- Errors per Key Pressed - number of times the backspace key is pressed, versus the keys pressed;

- Mouse Velocity - velocity at which the cursor travels;

- Mouse Acceleration - acceleration of the mouse at a given time;

- Time Between Keys - time spent between each two keys pressed;

- Total Excess of Distance - excess of distance travelled by the pointer when considering two consecutive clicks;

- Average Excess of Distance - average of the distance excess travelled by the pointer when considering two consecutive clicks;

- Double Click Speed - speed of the double click;

- Number of Double Clicks - number of double clicks in a time frame;

- Distance While Clicking - distance travelled by the mouse while dragging objects;

- Signed Sum of Angles - how much the pointer "turned" left or right during its travel;

- Absolute Sum of Angles - how much the pointer "turned" during its travel, in absolute terms;

- Sum of Distances between Path and Straight Line - considering two consecutive clicks, it measures the distance between all the points of the path travelled by the mouse, and the closest point in a straight line (that represents the shortest path) between the coordinates of the two clicks;

- Average Distance between Path and Straight Line - the same as above, but provides an average value of the distance to the straight line; and

- Time Between Clicks - time spent between each two clicks. 


\section{The Mental Fatigue Monitoring Framework}

As seen in section 2, indicators of mental fatigue are defined by a set of metrics. In order to have a positive and optimized effect on the lives of its users, a framework for fatigue monitoring based on these metrics must perform classification and decision-making in real-time.

The architecture of the proposed framework includes not only the sheer acquisition and classification of the data, but also a presentation tier that will support the human-based or autonomous decision-making mechanisms that are now being implemented. It is a layered architecture. The first layer is the Data acquisition one. It is responsible for capturing information describing the behavioral patterns of the user, receiving data from events fired from the use of the mouse and the keyword. Therefore, this layer encodes each event with the corresponding necessary information (e.g. timestamp, coordinates, type of click, key pressed).

The second layer is the Data processing. In this layer the data received from the Data acquisition layer is processed and transformed in order to be evaluated according to the metrics presented. One of the most important tasks of this layer is to filter outlier values that would have a negative effect on the analysis (e.g. a key pressed for more than a certain amount of time).

The next layer is the Classification layer. This layer is responsible for interpreting data from the mental fatigue indicators and to build the meta data that will support decision-making. To do it, this layer uses the machine learning mechanisms detailed below.

After the classification, the Data access layer, is responsible for providing access to - and persisting all the information generated by - the lower layer. We are not only interested in allowing access to the data in real-time but also to persist the historic of the user as well as his/her profile, to allow analyses within longer time frames.

Finally, the Presentation layer includes the mechanisms to build intuitive and visual representations of the mental states of the users, abstracting from the complexity of the data level where they are positioned.

\subsection{Classifying Fatigue}

The classification of the mental state of a user is achieved through the use of the k-Nearest Neighbor algorithm (k-NN). It is a method of classification based on closest training examples in the feature space. The data used to train the model used by the k-NN algorithm was collected in an experiment performed with students of the University of Minho, as described in section 4. From the available features, only the ones showing the most statistical significance were selected: Mouse Acceleration, Mouse Velocity, Keydown Time, Time between keys, and Error per key.

A different classifier is trained for each feature in order to get the level of fatigue due to each one. This is of significant importance since there are periods of time during which the framework only accesses a limited set of features (e.g. 
the user may not use the keyboard for a certain period of time). Thus, each feature is classified independently, assigning a binary value to each of its inputs, marking it as fatigued or not fatigued. Afterwards, a weighted sum is computed involving all the features with available data. The weights of the features for the overall computation of the level of fatigue are given by the significance of the feature, computed during the statistical analysis of the data at hand, as described in section 4 . This means that features that have been significantly affected by fatigue will have a larger contribution to the computed value than the ones that were hardly affected during the experiment. Using training data for a specific individual, when available, ensures that the fatigue detection model is personalized, thus more accurate.

The prototype of the presentation layer (Figure 1) uses a set of emoticons to depict the state of each user in an intuitive way that is easy to understand. For more detail it is also possible to access a more detailed interface, which shows not only the overall state of fatigue of the user (in a binary form as fatigued or not fatigued) but also the level of fatigue, which actually represents the weighted sum of the features.
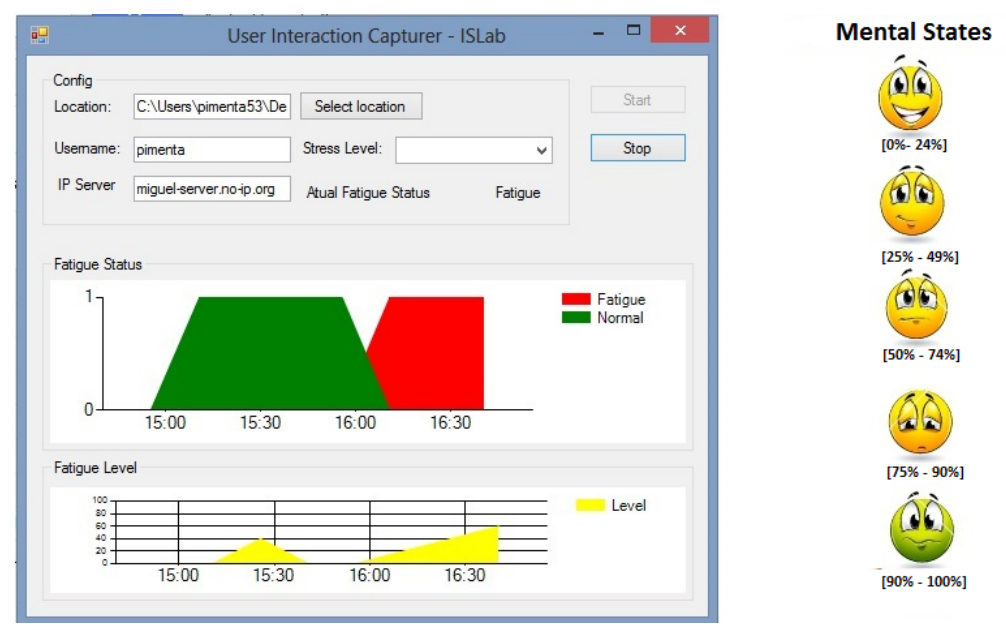

Fig. 1. The presentation layer of the monitoring system detailing the level of fatigue of an individual in part of the afternoon.

\section{Analysis of Results}

Twenty participants took part on the experiment in order to collect data to train the fatigue detection module. In this experiment participants needed only have an application running that recorded all their interactions with the keyboard and 
the mouse. It did not interfere with their usage patterns nor needed the participants to perform additional or different activities. The participants (seventeen males and three females) were mostly volunteer students from our institution, aged eighteen to fifty. All these individuals were familiar with the technological devices used and the interaction with them was not an obstacle.

The collection of the data took place in two moments, for each user: the first at the beginning of the day, where participants are fully rested and in a normal mental state and the second at the end of the day. The two two collection moments took place on the same day. It is also important to mention that the collection of data took place during the exam season and that the participants spent the day studying, with the support of a computer.

\subsection{Statistical data Analysis}

To determine to which extent each feature considered is or is not influenced by mental fatigue, the data collected in the first phase (without fatigue) is compared with the data collected in the second phase (with expected mental fatigue). Given that most of the distributions of the data collected are not normal, the Mann-Whitney test is used to perform the analysis. This test is a non-parametric statistical hypothesis test for assessing whether one of two samples of independent observations tends to have larger values than the other. The null hypothesis is thus: $H_{0}=$ the medians of the two distributions are equal. For each two distributions compared, the test returns a $p$-value, with a small $p$-value suggesting that it is unlikely that $H_{0}$ is true. Thus, for every Mann-Whitney test whose $p-$ value $<\alpha$, the difference is considered to be statistically significant, i.e., $H_{0}$ is rejected, with $\alpha=0.05$. The data analysis was performed using Wolfram Mathematica, Version 8.0.

A significant difference between the collected data in the two phases means that the feature is effectively influenced by mental fatigue for this specific individual. Statistically significant differences were observed in the features Keydown Time, Errors per Key Pressed, Time Between Keys, Mouse Velocity and Mouse Acceleration, hence only these are used for building the model.

For each feature and for each of the two moments of data collection, the average and median values were analyzed in order to determine the trend of the value, i.e., we wanted to answer the question "does it tend to decrease or to increase under fatigue?". Table 1 summarizes these observations.

The results obtained lead us to conclude that all the features have a significantly marked trend, either increasing or decreasing. The only exception to this is the feature Time between Clicks, which increased to half of the participants and decreased to the other half.

After the statistical analysis performed, one may claim that it is indeed possible to detect mental fatigue through the handling of keyboard and mouse usage data. It also proves, once more, that the presence of mental fatigue is accompanied by a loss of performance and an increase in errors, and that this loss may be measured using non-invasive tools. This loss of performance and increase in the number of errors are consequences of decreased cognitive skills, 


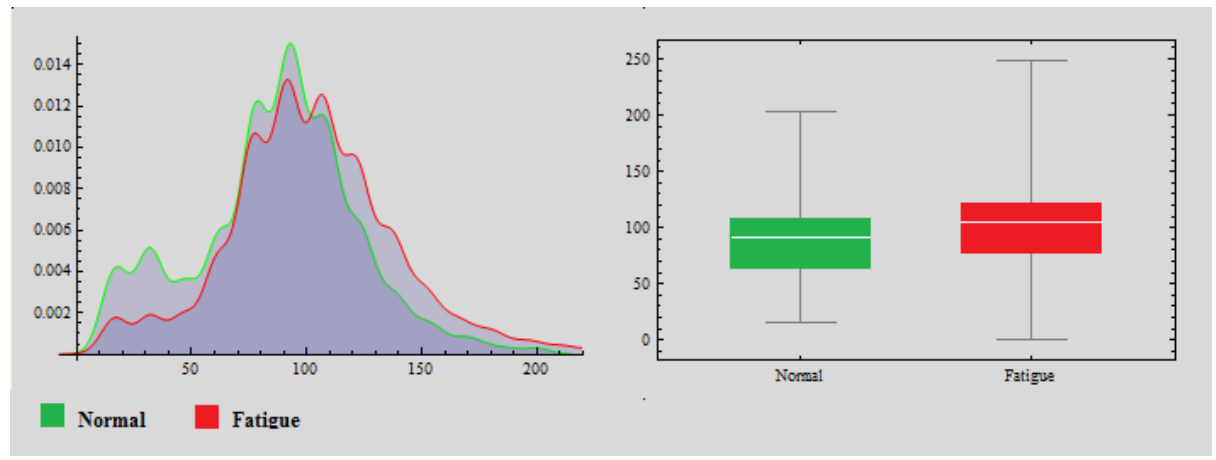

Fig. 2. Histograms and Box Plots comparing the data of the two distributions for the feature Keydown Time of a specific volunteer: fatigued individuals tend to write slower.

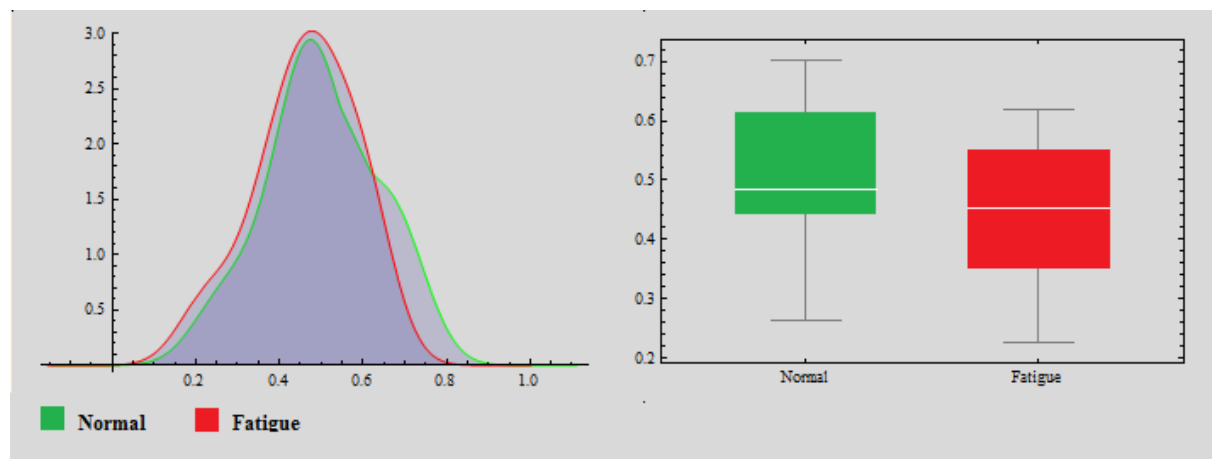

Fig. 3. Histograms and Box Plots comparing the distributions of the data collected in the two moments for the feature Mouse Velocity of a volunteer: fatigued individuals move the mouse slower.

which are caused by the increasing level of fatigue being measured during the day's work. The decrease in the cognitive skills is evidenced by general patterns of slower mouse (mouse acceleration and velocity) and keyboard (Keydown Time, Time between keys) interactions, as well as by an increasing number of errors (Error per key).

\section{Conclusion}

In this paper it is presented an approach to classify the level of mental fatigue of the individuals using a computer, by analyzing their interaction patterns, specifically the aspects related to the use of the mouse and the keyboard. The most noteworthy aspects of the work presented is that it details a non-invasive, nonintrusive and transparent approach to solve the problem. Existing approaches 
Table 1. Results of the statistical analysis of the data for the 20 participants. Only the features that have shown significant differences are included. The "trend" column depicts the percentage of participants that have a given trend. For example, the mean value of the velocity of the mouse decreases for $90 \%$ of the students, when fatigued.

\begin{tabular}{|l|l|l|l|l|l|}
\hline \multicolumn{2}{|c|}{ Metric } & Normal & Fatigued & Trend & p-value \\
\hline Keydown Time & Mean: & 79.827 & 87.119 & Increases in 100\% & $0.7 * 10^{-4}$ \\
& Median: & 77.601 & 81.502 & Increases in $60 \%$ & \\
\hline Time between keys & Mean: & 469.193 & 1040.26 & Increases in 100\% & $1.23 * 10^{-144}$ \\
& Median: & 215.75 & 386.55 & Increases in 90\% & \\
\hline Mouse Acceleration & Mean: & 0.4238 & 0.3829 & Decreases in 90\% & $3.01 * 10^{-11}$ \\
& Median: & 0.2202 & 0.2010 & Decreases in 100\% & \\
\hline Mouse Velocity & Mean: & 0.5002 & 0.4401 & Decreases in 90\% & $5.03 * 10^{-15}$ \\
& Median: & 0.2680 & 0.2537 & Decreases in 100\% & \\
\hline Time between Clicks & Mean: & 3081.35 & 3257.61 & Increases in 50\% & $5.8 * 10^{-4}$ \\
& Median: & 1733.30 & 1863.15 & Increases in 50\% & \\
\hline Error per key & Mean: & 7.643 & 9.002 & Increases in 90\% & $2 * 10^{-2}$ \\
& Median: & 7.444 & 8.598 & Increases in 90\% & \\
\hline
\end{tabular}

are either based on questionnaires or on physiological sensors, both with disadvantages of their own. The approach presented is based on the analysis of the user's behaviour. The results obtained prove not only the effect of fatigue on the user's performance throughout the day but also that it is possible to measure and classify these effects, in real time. This work opens the door to the development of leisure and working environments that are aware of their user's level of fatigue, and may, therefore, provide decision-support systems that will improve their working performance and their quality of life.

\section{Acknowledgements}

This work is part-funded by ERDF - European Regional Development Fund through the COMPETE Programme (operational programme for competitiveness) and by National Funds through the FCT - Fundação para a Ciência e a Tecnologia (Portuguese Foundation for Science and Technology) within project FCOMP-01-0124-FEDER-028980 (PTDC/EEI-SII/1386/2012). The work of Davide Carneiro is also supported by a doctoral grant by FCT (SFRH/BD/64890/2009).

\section{References}

1. van der Linden, D., Eling, P.: Mental fatigue disturbs local processing more than global processing. Psychological research 70(5) (September 2006) 395-402

2. Williamson, R.J., Purcell, S., Sterne, A., Wessely, S., Hotopf, M., Farmer, A., Sham, P.C.: The relationship of fatigue to mental and physical health in a community sample. Social psychiatry and psychiatric epidemiology 40(2) (February 2005) 126-32 
3. Nussbaum, M.A., Babski-reeves, K.L., Kleiner, B.M., Smith-jackson, T.L., Barker, L.M.: Measuring and modeling the effects of fatigue on performance : Specific application to the nursing profession Linsey Marinn Barker Virginia Polytechnic Institute and State University to partially fulfill the requirements of a Doctor of Philosophy In Indus. PhD thesis (2009)

4. Winwood, P.C., Winefield, a.H., Dawson, D., Lushington, K.: Development and Validation of a Scale to Measure Work-Related Fatigue and Recovery: The Occupational Fatigue Exhaustion/Recovery Scale (OFER). Journal of Occupational and Environmental Medicine 47(6) (June 2005) 594-606

5. Kobayashi, H., Demura, S.: Relationships between Chronic Fatigue, Subjective Symptoms of Fatigue, Life Stressors and Lifestyle in Japanese High School Students. School Health 2 (2006) 5

6. Joyce, E., Blumenthal, S., Wessely, S.: Memory, attention, and executive function in chronic fatigue syndrome. Journal of neurology, neurosurgery, and psychiatry 60(5) (May 1996) 495-503

7. Hossain, J.L., Ahmad, P., Reinish, L.W., Kayumov, L., Hossain, N.K., Shapiro, C.M.: Subjective fatigue and subjective sleepiness: two independent consequences of sleep disorders? Journal of sleep research 14(3) (September 2005) 245-53

8. Joyce, J., Rabe-Hesketh, S., Wessely, S.: Reviewing the reviews: the example of chronic fatigue syndrome. JAMA : the journal of the American Medical Association 280(3) (July 1998) 264-6

9. Neuberger, G.B.: Measures of fatigue: The Fatigue Questionnaire, Fatigue Severity Scale, Multidimensional Assessment of Fatigue Scale, and Short Form-36 Vitality (Energy/Fatigue) Subscale of the Short Form Health Survey. Arthritis \& Rheumatism 49(S5) (October 2003) S175-S183

10. Novais, P., Carneiro, D., Gomes, M., Neves, J.: Non-invasive Estimation of Stress in Conflict Resolution Environments. In Demazeau, Y., Müller, J.P., Rodríguez, J.M.C., Pérez, J.B., eds.: Advances on Practical Applications of Agents and Multi-Agent Systems. Volume 155 of Advances in Intelligent and Soft Computing. Springer Berlin Heidelberg (2012) 153-159

11. Carneiro, D., Castillo, J.C., Novais, P., Fernández-Caballero, A., Neves, J.: Multimodal Behavioural Analysis for Non-invasive Stress Detection. (July 2012) (2012) 